\title{
Strength at the Interface of CNT Films Made by Surface Decomposition of SiC
}

\author{
Yosuke Tsukiyama $^{1)^{*}}$, Noritsugu Umehara ${ }^{1)}$ and Michiko Kusunoki ${ }^{2)}$ \\ 1) Department of Mechanical Science and Engineering, Graduate School of Engineering, Nagoya University \\ Furo-cho Chikusa-ku, Nagoya, Aichi 484-8603, Japan \\ ${ }^{2)}$ Division of Environmental Research, EcoTopia Science Institute, Nagoya University \\ Furo-cho Chikusa-ku, Nagoya, Aichi 484-8603, Japan \\ *Corresponding author: tsukiyama@nagoya-u.jp
}

( Manuscript received 2 September 2008; accepted 29 October 2008; published 31 December 2008 )

(Presented at the International Session, JAST Tribology Conference Nagoya, September 2008 )

\begin{abstract}
Adherence of carbon nanotube film made by surface decomposition of $\mathrm{SiC}$ was studied. For this purpose we carried out scratch tests in nano- and microscale. By microscratch test, it was found that adherence at the interface was higher for CNT films with shorter CNTs and CNTs were not broken at the middle but removed from the interface. To clarify the dominant force (shear force or moment of force) which caused different adherence, we also carried out nanoscratch test by using AFM. In nanoscratch, AFM tip made of diamond coated silicon applied shear force to CNT films from lateral direction. And there was no significant change of adherence by lateral force (shear force at the interface). We conclude that bending-induced moment of force is dominant on the interface strength of CNT films.
\end{abstract}

Keywords: carbon nanotube, interface, scratch, AFM

\section{Introduction}

Since the discovery of carbon nanotube $(\mathrm{CNT})^{1)}$, its mechanical and structural properties have attracted much attention. For the purpose of macro scale applications of this nanometer scale material, some methods such as chemical vapor deposition (CVD) have been shown for vertically aligned CNT films ${ }^{2,3}$. And some tribological properties of CNT films have been also studied ${ }^{4-6)}$ Regarding the CNT films as new carbon coatings for tribosystems, it is necessary to clarify the adherence of CNT films against the substrates. Despite of the importance, there are few studies showing the interface strength of CNT films. As for adherence of CNT films, bonds between CNTs and substrates play an important role for determining the interfaces' strength.

Kusunoki et al. have reported that vertically aligned and densely packed carbon nanotubes were grown on Silicon carbide $(\mathrm{SiC})$ substrates by annealing in a vacuum chamber ${ }^{7,8)}$. Compared with other synthesis methods such as CVD, this method enables one to make CNT films having strong adherence due to covalent bonds of CNTs to SiC substrates. Although many papers have reported about the strength and elastic behavior of CNT itself ${ }^{9,10)}$, few studies have been reported about interface strength of CNT film. Therefore the purpose of this study is to clarify the strength at the interface of CNT film made by surface decomposition of $\mathrm{SiC}$. For this purpose, we carried out scratch tests in a couple of scales (micro and nano scale).

\section{Experiment}

2.1 Specimen

We prepared CNT films by surface decomposition of $\mathrm{SiC}$. CNTs were grown only from the C-face of commercial $6 \mathrm{H}-\mathrm{SiC}$ substrates under $1700{ }^{\circ} \mathrm{C}$ in a vacuum chamber. The length of CNTs increases with annealing time. The synthesis is shown elsewhere in more detail ${ }^{7)}$. As shown in Table 1, CNT films with CNT length of 25, 50, 100 and $210 \mathrm{~nm}$ and with uniform diameter of $5 \mathrm{~nm}$ (average) were prepared.

Table 1 Specimens

\begin{tabular}{|c|c|c|c|c|c|}
\hline Specimen & CNT1 & CNT2 & CNT3 & CNT4 & CNT5 \\
\hline Length & $10 \mathrm{~nm}$ & $25 \mathrm{~nm}$ & $50 \mathrm{~nm}$ & $100 \mathrm{~nm}$ & $210 \mathrm{~nm}$ \\
Diameter & $5 \mathrm{~nm}$ & $5 \mathrm{~nm}$ & $5 \mathrm{~nm}$ & $5 \mathrm{~nm}$ & $5 \mathrm{~nm}$ \\
\hline
\end{tabular}

2.2 Experimental apparatus and Methods

We carried out microscratch test and nanoscratch test. The purpose was to measure the critical loads when CNT films were removed by the tips. Table 2 shows the comparison of the tips used in these tests. The estimated contact area under the critical load was nanometer scale 
and micrometer scale with respect to nanoscratch and microscratch.

Figure 1 shows the schematic of the microscratch test. We used a commercial scratching apparatus (RHESCA). In this apparatus, the cartridge was oscillated in a perpendicular direction ( $\mathrm{x}$ direction in Fig 1) to scratch direction (y direction in Fig. 1). So the tip on a cantilever was also oscillated with the cartridge and the oscillating amplitude at the tip was $80 \mu \mathrm{m}$. And the stage was inclined with the angle of 5 degrees to horizontal plane in order to gradually increase normal load as a stage motion. When a film is removed under critical load, debris-derived change in a friction coefficient causes change in voltage by electromagnetic induction between a magnet and a coil. The stage speed was approximately $20 \mu \mathrm{m} / \mathrm{sec}$.

Nanoscratch tests were carried out by using Atomic Force Microscope (AFM). We used commercial AFM (SPA400 and SPI3800 N, SII Inc.) and normal load was controlled to be constant during each scratch. First, CNT stripes with various width of $0.2,0.4,0.6,0.8,1.0$ $\mu \mathrm{m}$ were made by nanoscratch applying an enough load to remove CNTs (Fig. 2a, b). After that, the tip scratched perpendicularly to the stripes (Fig. 2c). The tip applied the lateral force to $\mathrm{CNT}$ films from removed part of CNT films. Comparing with the normal contacts between a tip and surfaces in micro-scratch tests (usual type of contact with a sphere and a plane), the lateral contacts of AFM tips in nanoscratch tests apply shear force to the interface between CNT films and $\mathrm{SiC}$ substrates directly. After measuring the critical loads in both tests we observed the corresponding parts of specimens by FE-SEM in order to confirm removal of CNTs.

The effect of wear of tips was thought to be negligible because of repeatability of results for several times.

Table 2 Comparison of the tips used in nanoscratch and microscratch test

\begin{tabular}{lll}
\hline & Tip radius & Tip material \\
\hline $\begin{array}{l}\text { Nano- } \\
\text { scratch }\end{array}$ & $100 \mathrm{~nm}$ & Diamond coated Silicon \\
$\begin{array}{l}\text { Micro- } \\
\text { scratch }\end{array}$ & $5 \mu \mathrm{m}$ & Diamond \\
\hline
\end{tabular}

\section{Results and discussion}

Figure 3 shows the critical loads of CNT films as a function of CNT length by microscratch test.

Noting that the vertical axis is log scale, there are significant differences in critical loads (adherence). Shorter CNT films showed much higher adherence than longer CNT film. The Hertz contact pressure was more than $30 \mathrm{GPa}$ under critical load for 10 and $25 \mathrm{~nm}$

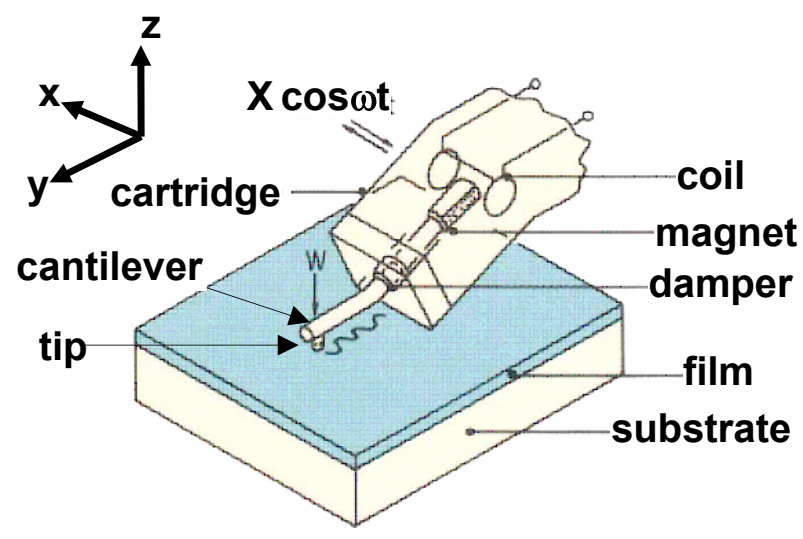

Fig. 1 Schematic of microscratch test

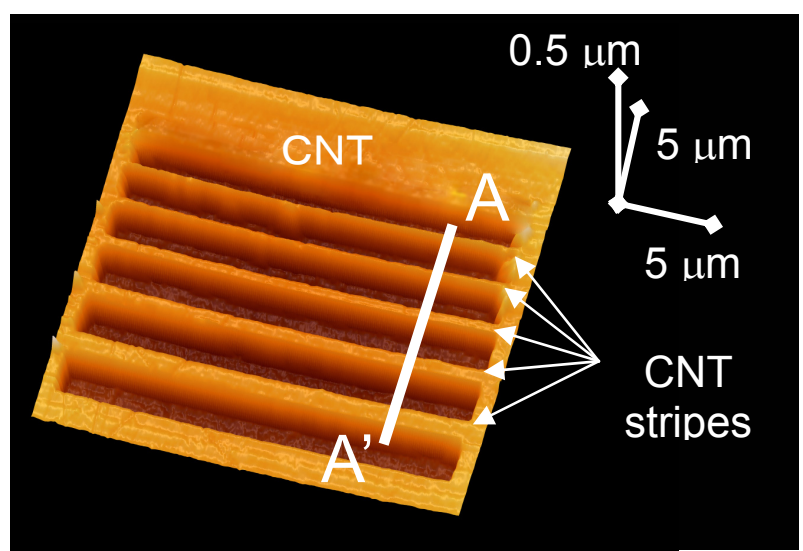

(a) AFM image of CNT stripes

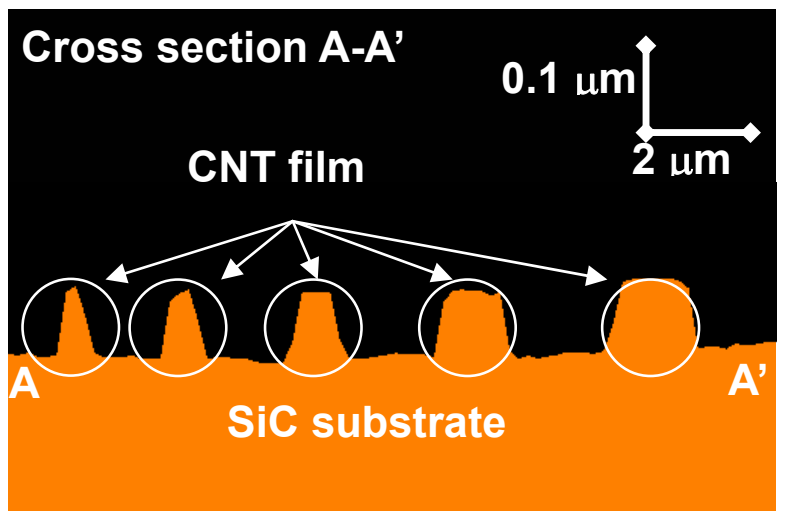

(b) Cross section of CNT stripes

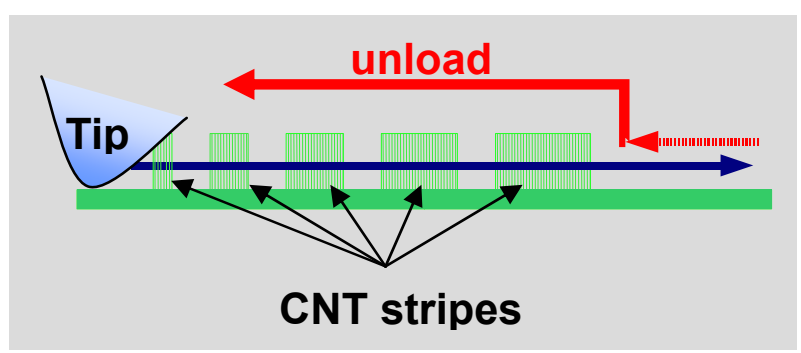

(c) Nanoscratch of CNT stripes

Fig. 2 Schematic of nanoscratch test 
CNT film. Figure 4 shows the surface profile of the scratched area by using AFM. In the initial stage of scratch, one can see the scratch trace stretching for approximately $15 \mu \mathrm{m}$ along scratch direction, and then, CNT removal occurred (Fig. 4a). CNTs were removed from their roots and not broken at the middle (Fig. 4b).

Figure 3 clearly shows the dependence of adherence on CNT length. Here, we assumed that strength at the interface of CNT films is independent of the CNT length correlating to annealing time during the surface decomposition method, because no defects were found in each CNTs observed by high-resolution transmission electron microscopy ${ }^{8}$. Based on this assumption, the adherence of CNT films can be affected by bending-induced moment of force at the root of each CNTs and/or shear force at the interfaces. To clarify the dominant force which causes removal of CNTs, we carried out nanoscratch tests.

As shown before, nanoscratch by AFM applies force against CNT stripes in lateral direction. Although the scratch tests used programmed trajectories for the same experimental condition, there were influence and dependence of CNT length on normal and lateral critical loads (Fig. $5 \mathrm{a}$ and b). The observation by FE-SEM after scratch tests showed that the area of removed CNTs by one scratch was not same as that of other scratches with different loads or CNT lengths. FE-SEM observation of the scratched areas enabled us to measure the removal area of CNT stripes. And we derived the lateral force per unit removal area (lateral stress, $\sigma$ ) shown in Fig. 6.

The lateral stress under critical load was proportional to the inverse of CNT length. By a linear model of an elastic bar in which the lateral stress is homogeneously distributed on the side surface of a CNT, the moment of force $M$ is written as Eq. (1), where L is CNT length and $a$ is cross-section area of a CNT. And this means lateral stress is proportional to the moment of force and the inverse of CNT length. As mentioned above, the maximum moment of force, the strength at the root of CNT, is constant because CNT films have adherence independent on CNT length. Therefore the lateral stress is written by using constant number $C$ as Eq. (2).

$$
\begin{aligned}
& M=1 / 2 \cdot a \cdot L \cdot \sigma \\
& \sigma=C L^{-1}
\end{aligned}
$$

Equation (2) matches the experimental result shown in Fig. 6. Based on this result, we conclude that the bending-induced moment of force at the root of each CNT is dominant on the interface strength of CNT films rather than shear force.

\section{Conclusion}

We carried out nano- and microscratch tests to clarify the interface strength of CNTs. In microscratch

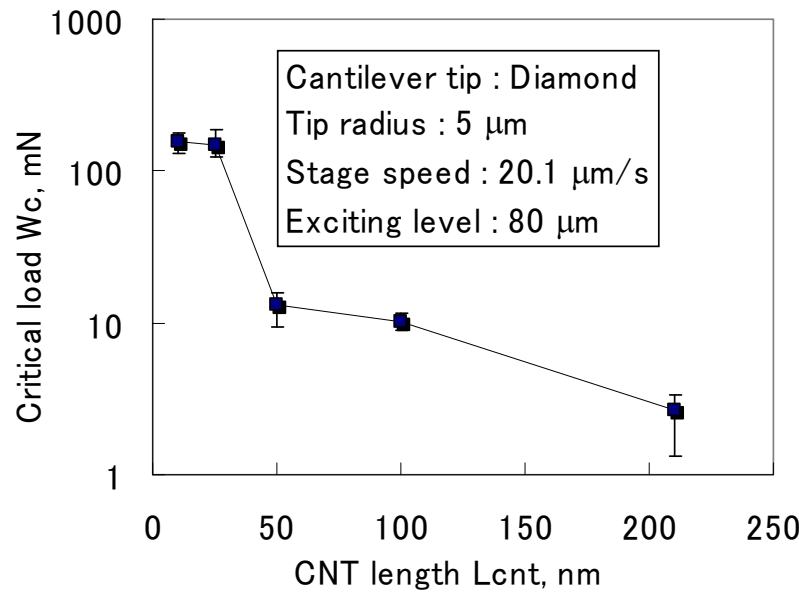

Fig. 3 Critical loads of CNT films by micro-scratch

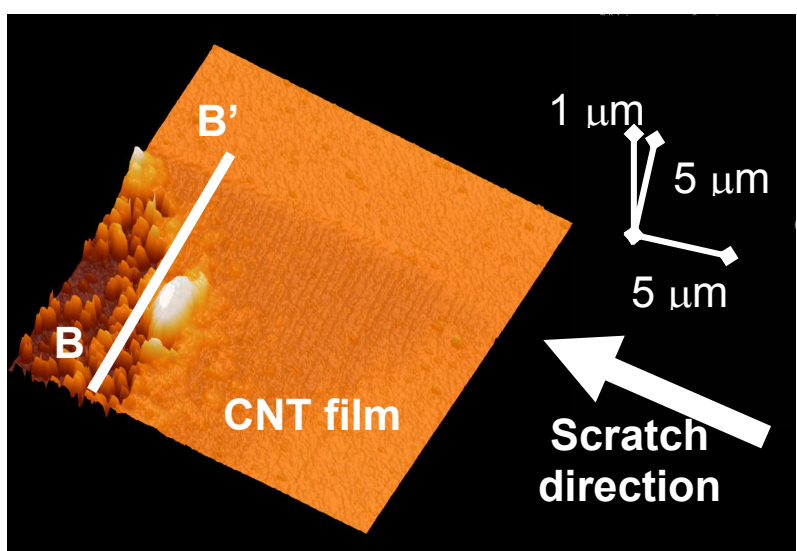

(a) Surface profile of CNT film where CNT removal began

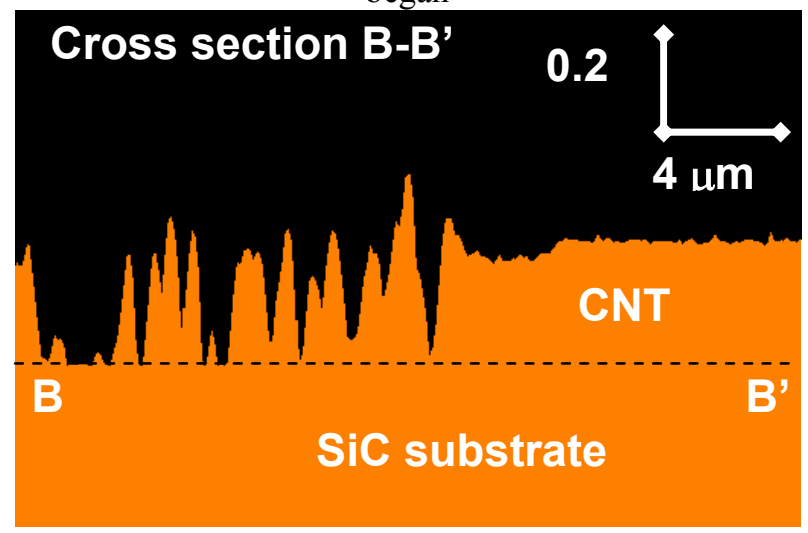

(b) Cross section of scratched area

Fig. 4 AFM image of scratched CNT (200 nm long) film after microscratch

test, the CNT films showed significant difference in critical load among specimens with different length of CNT. And shorter CNT films showed much higher critical load than longer CNT films.

Especially, 10 and $25 \mathrm{~nm}$ CNT showed high adherence under the critical load deriving contact pressure of more than $30 \mathrm{GPa}$. 


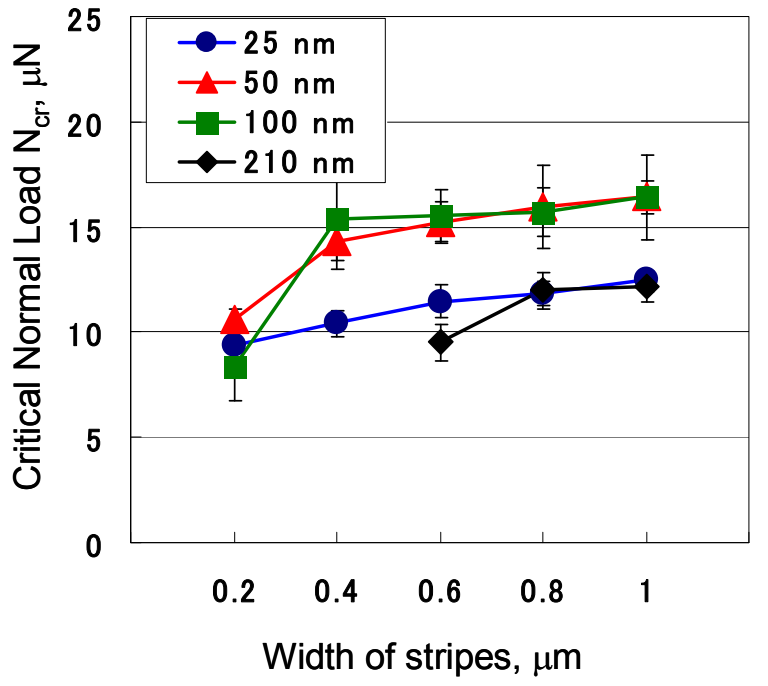

(a) Critical normal load

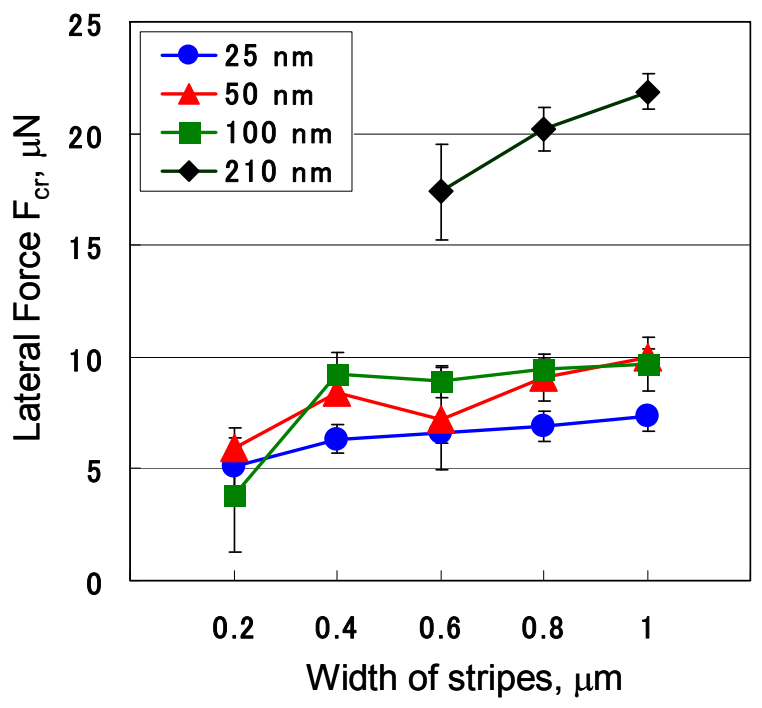

(b) Lateral force

Fig. 5 Critical loads of nanoscratch test

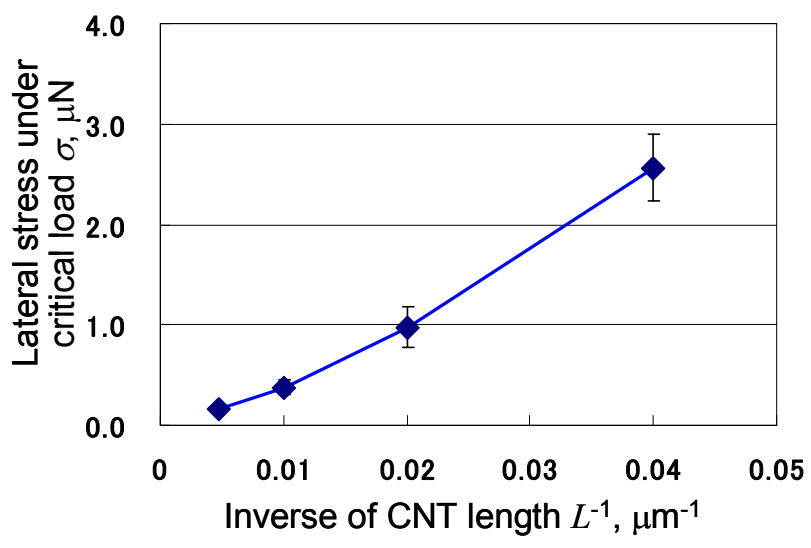

Fig. 6 Lateral stress under critical load as a function of CNT length
By using AFM, nanoscratch test showed that lateral stress under critical load was proportional to the inverse of CNT length meaning bending-induced moment of force is dominant on interface strength rather than shear force.

\section{References}

[1] Iijima, S., "Helical Microtubules of Graphitic Carbon," Nature, 354, 1991, 56-58.

[2] Li, W. Z., Xie, S. S., Qian, L. X., Chang, B. H., Zou, B. S., Zhou, W. Y., Zhao, R. A. and Wang, G., "Large-Scale Synthesis of Aligned Carbon Nanotubes," Science, 274, 1996, 1701-1703.

[3] Terrones, M., Grobert, N., Olivares, J., Zhang, J. P., Terrones, H., Kordatos, K., Hsu, W. K., Hare, J. P., Townsend, P. D., Prassides, K., Cheetham, A. K., Kroto, H. W. and Walton, D. R. M., "Controlled Production of Aligned-Nanotube Bundles," Nature, 388, 1997, 52-55.

[4] Kinoshita, H., Kume, I., Tagawa, M. and Ohmae, N., "High Friction of a Vertically Aligned Carbon-Nanotube Film in Microtribology," Applied Physics Letters, 85, 2004, 2780-2781.

[5] Miyake, K., Kusunoki, M., Usami, H., Umehara, N. and Sasaki, S., "Tribological Properties of Densely Packed Vertically Aligned Carbon Nanotube Film on SiC Formed by Surface Decomposition," Nano Letters, 7, 2007, 3285-3289.

[6] Turq, V., Ohmae, N., Martin, J. M., Fontaine, J., Kinoshita, H. and Loubet, J., "Influence of Humidity on Microtribology of Vertically Aligned Carbon Nanotube Film," Tribology Letters, 19, 2005, 23-28.

[7] Kusunoki, M., Rokkaku, M. and Suzuki, T., "Epitaxial Carbon Nanotube Film Self-Organized by Sublimation Decomposition of Silicon Carbide," Applied Physics Letters, 71, 1997, 2620-2622.

[8] Kusunoki, M., Suzuki, T., Kaneko, K. and Ito, M., "Formation of Self-Aligned Carbon Nanotube Films by Surface Decomposition of Silicon Carbide," Philosophical Magazine Letters, 79, 1999, 153-161.

[9] Yu, M. F., Lourie, O., Dyer, M. J., Moloni, K., Kelly, T. F. and Ruoff, R. S., "Strength and Breaking Mechanism of Multiwalled Carbon Nanotubes under Tensile Load," Science, 287, 2000, 637-640.

[10] Demczyk, B. G., Wang, Y. M., Cumings, J., Hetman, M., Han, W., Zettl, A. and Ritchie, R. O., "Direct Mechanical Measurement of the Tensile Strength and Elastic Modulus of Multiwalled Carbon Nanotubes," Materials Science and Engineering A-Structural Materials Properties Microstructure and Processing, 334, 2002, 173-178. 